\title{
Serogroups and virulence genotypes of Escherichia coli isolated from patients with sepsis
}

\author{
M. Ananias and T. Yano \\ Laboratório de Fatores de Virulência em Bactérias, Departamento de Microbiologia e Imunologia, \\ Instituto de Biologia, Universidade Estadual de Campinas, Campinas, SP, Brasil \\ Correspondence to: T. Yano, Departamento de Microbiologia e Imunologia, IB, UNICAMP, 13083-862 \\ Campinas, SP, Brasil \\ Fax: +55-19-3521-6276. E-mail: tyano@unicamp.br
}

Sixty strains of Escherichia coli, isolated by hemoculture, from septicemic Brazilian patients were evaluated to determine their serogroup and invasivity to Vero cells. All 60 patients died within 2 days of hospitalization. Furthermore, the molecular study of the following extraintestinal pathogenic $E$. coli-associated virulence factor (VF) genes was performed by PCR: i) adhesins: type 1 fimbria $(f i m H)$, S fimbria ( $s f a D / E)$, P fimbria ( $p a p C$ and $p a p G$ alleles) and afimbrial adhesin (afaB/C); ii) capsule K1/K5 (kpsMTII); iii) siderophores: aerobactin (iucD), yersiniabactin (fyuA) and salmochelin (iroN); iv) toxins hemolysin ( $h l y A)$, necrotizing cytotoxic factor type 1 (cnf1) and secreted autotransporter toxin (sat); v) miscellaneous: brain microvascular endothelial cells invasion (ibeA), serum resistance (traT), colicin $\mathrm{V}(\mathrm{cvaC})$ and specific uropathogenic protein (usp). Our results showed that isolates are able to invade Vero cells (96.6\%), differing from previous research on uropathogenic $E$. coli (UPEC). The $O$ serogroups associated with UPEC were prevalent in $60 \%$ of strains vs $11.7 \%$ of other serogroups. The PCR results showed a conserved virulence subgroup profile and a prevalence above $75 \%$ for fimH, fyuA, kpsMTII and iucD, and between $35-$ $65 \%$ for papC, papG, sat, iroN, usp and traT. The evasion from the immunological system of the host and also iron uptake are essential for the survival of extraintestinal pathogenic $E$. coli strains. Interestingly, among our isolates, a low prevalence of VF genes appeared. Therefore, the present study contributes to the identification of a bacterial profile for sepsis-associated $E$. coli.

Key words: Escherichia coli; Sepsis; Serogroups; Virulence genotypes

Publication supported by FAPESP.

Received December 3, 2007. Accepted September 3, 2008

\section{Introduction}

Pathogenic Escherichia coli strains cause intestinal or extraintestinal infections in many host species and they are the most frequent cause of Gram-negative extraintestinal infections, including urinary tract infections, newborn meningitis, and septicemia (1). Several virulence factors (VFs) enhance the capacity of $E$. coli to cause systemic infections; unlike most commensal E. coli strains, extraintestinal isolates possess genes encoding putative VFs of extraintestinal pathogenic E. coli (ExPEC), which include various combinations of adhesins ( $P$ and $S$ fimbriae), toxins (e.g., hemolysin and cytonecrotizing factor), polysaccharide coatings (including capsules and lipopolysaccharides), siderophores or iron acquisition systems (e.g., aerobactin and yersiniabactin), serum resistance mechanisms, and invasins (2). These VFs help the organisms to colonize host surfaces, avoid and/or subvert host defense mechanisms, injure and/or invade host cells and tissues, and incite a noxious inflammatory response, thereby leading to clinical disease (2). The ExPEC VF genes are thought to be primarily inherited vertically within evolutionary lineages and also to be transferred horizontally between lineages, in some instances on plasmids or on 
"pathogenicity-associated islands" (PAls), which are gene blocks that contain multiple contiguous VF genes (2). The prevalence and evolutionary origins of the ExPEC VFs and of the distinctive "virulent clones" that make up the ExPEC population require a better understanding to hasten the development of preventive measures that are sorely needed against these morbid and costly infections (3). Moreover, ExPEC strains constitute an increasing problem for human medicine, especially due to the high incidence of drug resistance often transmitted by plasmids. In addition, to human diseases, ExPEC strains are the cause of significant economic losses on animal farms, especially in the poultry industry. Neither the VFs of ExPEC strains nor the differences between the virulence mechanisms of the various pathogenic serotypes have yet been fully identified (1).

In this study, we determined the virulence gene distribution of sixty $E$. coli blood strains, which were isolated from Brazilian patients with sepsis. The serogrouping and the measurement of the ability of these cells to invade cells of kidney African green monkey (Vero) were also performed. Among the ExPEC, the genetic backgrounds of UPEC (uropathogenic E. coli) and APEC (avian pathogenic $E$. coli) are interestingly related to sepsis-associated E. coli (SEPEC), but consistent differences are evident. SEPEC seems to have a combination of VFs that are constant in UPEC and APEC, but a genetic profile for SEPEC has not yet been identified. The infectious mechanism is quite unique when comparing UPEC, APEC and SEPEC, since it ranges from singular anatomical sites to specific hosts. This suggests the existence of an individual group for SEPEC. To confirm this, it is necessary to identify a precise bacterial profile and an infectious mechanism, and neither has yet been elucidated. The present study increases the knowledge about this possible ExPEC group and provides the first data about SEPEC in Brazil.

\section{Material and Methods}

Sixty E. coli strains were isolated by blood culture from 60 adults with sepsis from 1998 to 2001 in the Laboratório de Patologia Clínica (Seção de Microbiologia Clínica), Hospital de Clínicas, UNICAMP, Campinas, SP, Brasil. E. coli FBC114, FV35 FVL2, FVL8, J96, U9-41 (all samples were kindly provided by Dr. Domingos da Silva Leite, UNICAMP), ORN115 (kindly provided by Dr. Paul E. Orndorff, North Carolina State University, USA), APEC (kindly provided by Dr. Marilda Carlos Vidotto, UEL, Londrina, PR, Brasil), and APEC colV+ (kindly provided by Dr. Wanderley Dias da Silveira, UNICAMP) were used as control strains. Clinical data from patients (Schreiber AZ, unpublished data) are totally heterogeneous: age groups, both genders, health and host compromise status (i.e., underlying medical conditions such as diabetes mellitus, cancer, immunosuppression, uremia or underlying urological conditions such as pre-existing urinary tract abnormalities, urinary tract instrumentation or pregnancy). All 60 septicemic patients died within 2 days of hospitalization with the diagnosis of sepsis. However, the study utilized $E$. coli isolates without the knowledge that the patients had died. Clinical data for these patients are incomplete, but mortality was attributable to, or associated with, E. coli.

All isolates were serogrouped according to Laboratorio de Referencia de E. coli (Universidad de Santiago de Compostela, Campus de Lugo, Lugo, Galicia, España) (http://www.lugo.usc.es/ecoli/) and $\mathrm{O}$ antisera were kindly supplied by Dr. D.S. Leite (UNICAMP).

The molecular biology study was performed by PCR of the following ExPEC-associated VF genes: i) adhesins: type 1 fimbria $(f i m H)$ (4), S fimbria (sfaD/E) (5) and $P$ fimbria $(p a p C)(5)$, afimbrial adhesin $(a f a B / C)(6)$, and the alleles I, II and III of the P fimbrial adhesin (papG) (7); ii) capsule K1/K5 (kpsMTII) (7); iii) siderophores: aerobactin (iucD) (6), yersiniabactin (fyuA) (8) and salmochelin (iroN) (4); iv) toxins: hemolysin ( $h l y A)(6)$, necrotizing cytotoxic factor type 1 (cnf1) (6) and toxin autotransporter secreted (sat) (present study); v) miscellaneous: brain microvascular endothelial cells invasion (ibeA) (9), serum resistance (traT) (10), colicin V (cvaC) (10) and specific uropathogenic protein (usp) (11). For sat, we developed the following PCR primers: 5'GTTGTCTCTGGCTGTTGC3' and 5'AATGATGTTCCTCCAGAGC3' with 501-bp amplicon size, annealing temperature of $53^{\circ} \mathrm{C}$, and $\mathrm{FBC} 114$ strain as gene control.

Invasion was assessed using the gentamicin protection assay on the Vero cell line. Antibiotic sensitivity for gentamicin was tested using a disk diffusion test following the National Committee on Clinical Laboratory Standards (NCCLS) guidelines. Overnight bacterial cultures $\left(37^{\circ} \mathrm{C}\right)$ were resuspended in culture medium (Dulbecco's minimum essential medium, DMEN, Gibco, USA) supplemented with $10 \%$ fetal calf serum (FCS, Gibco) and 1\% nonessential amino acids (Gibco). Confluent monolayers of Vero cells in a 96-well plate were exposed to 107 colonyforming units (CFU) of different $E$. coli isolates. The plate was centrifuged for $10 \mathrm{~min}$ at $37^{\circ} \mathrm{C}(350 \mathrm{~g})$ and incubated at $37^{\circ} \mathrm{C}$ in a $5 \% \mathrm{CO}_{2}$-enriched atmosphere for $1 \mathrm{~h}$. The cells were rinsed five times with Hanks' balanced salt solution (HBSS, Gibco) and incubated for another hour at $37^{\circ} \mathrm{C}$ and $5 \% \mathrm{CO}_{2}$ in culture medium supplemented with 50 $\mu \mathrm{L} / \mathrm{mL}$ gentamicin (Gibco). Thereafter, the cells were rinsed five times with HBSS. Cell lysis was obtained by incubation 
for $10 \mathrm{~min}$ at room temperature with $1 \%$ Triton $\mathrm{X}-100$ in distilled water. Finally, $120 \mu \mathrm{L}$ of 10 -fold dilutions was placed on blood and MacConkey agar plates. After incubation for $24 \mathrm{~h}$ at $37^{\circ} \mathrm{C}$, the presence of colonies indicated invasion. This assay was performed in triplicate (10).

The hemolytic activity was assayed (triplicate) on $5 \%$ human blood agar plates. Blood was donated by healthy volunteers. All isolates were cultured using an inoculating needle for agar deeps and an inoculating loop for the agar plate and were incubated at $37^{\circ} \mathrm{C}$ for $24 \mathrm{~h}$. All strains were compared to a positive ( $E$. coli J96) - halo formation - and a negative (E. coli $\mathrm{DH} 5 \alpha)$ control.

\section{Results}

The $\mathrm{O}$ serogroups associated with UPEC $(\mathrm{O} 1, \mathrm{O} 2, \mathrm{O} 4$, 06, 07, 08, 09, 011, 012, 014, 015, 016, 017, 018, $\mathrm{O} 21, \mathrm{O} 22, \mathrm{O} 25, \mathrm{O} 50, \mathrm{O} 75, \mathrm{O} 77, \mathrm{O} 78, \mathrm{O} 81, \mathrm{O} 83, \mathrm{O} 85$, and O86) were prevalent in $60 \%$ of strains vs $11.7 \%$ of other serogroups. A large number $(28.3 \%)$ could not be assigned to a serogroup because they were not identifiable with standard antisera or were rough. Thus, of the 60 isolates, only $71.7 \%$ (43 isolates) could be classified using standard antisera. Eighteen serogroups were represented (01, 02, O4, O6, 07, 011, O16, 017, 019, O20, O25, O75, O78, O86, O99, O141, 0153 and O173) and nine of these harbored a unique strain $(\mathrm{O} 4, \mathrm{O} 16, \mathrm{O} 20, \mathrm{O} 78, \mathrm{O} 86$, 099, 0141, 0153, and 0173). Serogroups containing 5\% or more of the identifiable isolates were O2 (13.3\%), O17 (11.6\%), O6 (10\%), and 075 (5\%).

Among the $60 \mathrm{E}$. coli blood isolates from patients with sepsis, the various VF prevalence ranged from $6.7 \%$ [afaB/C (afimbrial adhesin) and $i b e A$ (invasion of brain endothelium)] to 95\% [ fimH (type 1 fimbria]); Table 1]. The papG allele I was not detected. Overall, the seven most prevalent VFs, in order of descending frequency were: fimH (95\%), kpsMTII and fyuA (81.7\%), iucD (78.3\%), papC (65\%), sat (55\%) and traT (50\%). Among papC $^{+}$strains, the highest allele prevalence to papG was II (61.5\%); but the allele III (20.5\%) presented higher numbers in association with other VFs. Individual profiles for each strain are given in Table 2.

All 60 E. coli strains tested were gentamicin-sensitive. Only two strains, 16 and 32 , were not able to invade the Vero patients with sepsis. cells. The remaining 58 strains could. However, our objective in this essay was only qualitative, e.g., we did not analyze our results in a quantitative manner. However, our results covered broad range, from a couple few to hundreds.

Fifteen (25\%) of our 60 isolates were positive for a hemolytic reaction. Similarly, the hly operon that encodes the $\alpha$-hemolysin was detected in $24.4 \%$ of isolates by PCR. However, three strains were discrepant compared to our genotypic and phenotypic results. Two of three produced the hemolytic halo, but they were PCR-negative and only one strain was phenotypically negative, but PCRpositive to hlyA operon.

\section{Discussion}

This is the first study characterizing $E$. coli isolates molecularly obtained in Brazil from septicemic patients based on the most common VF genes among ExPEC. We also provided $O$ serogrouping for our 60 isolates and determined invasivity to Vero cells. Studies showing invasivity of septicemic E. coli are rare. In contrast with the well-characterized diarrheagenic E. coli, or even UPEC, SEPEC does not have a defined molecular profile or a clear infectious mechanism. SEPEC emerges as an individual or peculiar $E$. coli group that seems to combine characteristics of other groups, such as diarrheagenic $E$. coli, APEC and UPEC. Choosing these three targets:

Table 1. Frequency of virulence factor (VF) genes in 60 Escherichia coli isolates from

\begin{tabular}{llcc}
\hline VF gene & Description & Total $(\mathrm{N}=60)$ & $\%$ \\
\hline fimH & Type 1 fimbria, adhesive subunit & 57 & $95.0 \%$ \\
fyuA & Yersiniabactin receptor & 49 & $81.7 \%$ \\
kpsMTII & Capsule & 49 & $81.7 \%$ \\
iucD & Aerobactin siderophore biosynthesis & 47 & $78.3 \%$ \\
papC & P fimbria assembly, outer membrane usher & 39 & $65.0 \%$ \\
sat & Secreted autotransporter toxin & 33 & $55.0 \%$ \\
traT & Outer membrane protein gene; surface & 30 & $50.0 \%$ \\
& exclusion; serum resistance & & \\
iroN & Catecholate siderophore receptor gene & 22 & $36.7 \%$ \\
usp & Uropathogen-specific protein & 22 & $36.7 \%$ \\
hlyA & Hemolysin toxin protein & 14 & $23.3 \%$ \\
cnf1 & Cytotoxic necrotizing factor 1 & 13 & $21.7 \%$ \\
sfaD/E & S fimbria & 11 & $18.3 \%$ \\
cvaC & Colicin V precursor & 8 & $13.3 \%$ \\
afaB/C & Dr-binding adhesions & 4 & $6.7 \%$ \\
ibeA & Invasion of brain endothelium A & 4 & $6.7 \%$ \\
papGII & P fimbria adhesin (alleles I, II and III) & $24^{*}$ & $61.5 \%$ \\
papGIII & & $8^{*}$ & $20.5 \%$ \\
papGII, III & & $5^{*}$ & $12.8 \%$ \\
\hline
\end{tabular}

${ }^{*}$ Total: $\mathrm{N}=39$ ( $\mathrm{P}$ fimbria adhesins were tested only for the $39 \mathrm{papC}^{+}$strains). 
Table 2. Serogroups $O$ and virulence factor (VF) genes of the 60 strains of Escherichia coli isolates from patients with sepsis.

\begin{tabular}{|c|c|c|c|c|c|c|c|c|c|c|c|c|c|c|c|c|c|c|}
\hline $\begin{array}{l}\text { Strain } \\
\text { number }\end{array}$ & $\begin{array}{l}\text { O Sero- } \\
\text { groups }\end{array}$ & Hly & $\begin{array}{l}\text { kps } \\
\text { MTII }\end{array}$ & $\begin{array}{l}\text { sfa } \\
D / E\end{array}$ & papC & papG & $\mathrm{fimH}$ & $a f a B / C$ & $i u c D$ & fyuA & iroN & hlyA & sat & cnf1 & $i b e A$ & $\operatorname{tra} T$ & cvaC & usp \\
\hline 1 & O6 & + & + & + & + & 3 & + & - & - & + & + & + & - & + & - & - & - & + \\
\hline 2 & O75 & + & + & + & + & 2 & + & - & + & + & + & + & + & - & - & - & - & + \\
\hline 3 & O7 & - & + & - & + & 2 & + & - & + & + & - & - & + & - & - & - & - & - \\
\hline 4 & $\mathrm{NI}$ & - & + & - & + & 2 & + & - & + & + & - & - & + & - & - & + & - & - \\
\hline 5 & O7 & - & + & - & + & 2 & + & - & + & + & - & - & + & - & - & - & - & - \\
\hline 6 & 0141 & - & + & - & + & 2 & + & - & + & + & - & - & + & - & - & - & - & - \\
\hline 7 & $\mathrm{O} 4$ & + & + & + & + & 3.2 & + & - & + & + & - & - & + & - & - & - & - & - \\
\hline 8 & O99 & - & + & - & - & NA & + & - & - & + & - & - & - & - & - & + & - & - \\
\hline 9 & $\mathrm{O} 2$ & - & + & + & + & 3.2 & + & - & + & + & + & + & + & + & - & + & - & + \\
\hline 10 & 011 & - & + & - & + & 2 & + & - & + & + & - & - & + & - & - & + & - & - \\
\hline 11 & O6 & + & + & + & + & 3 & + & - & - & + & + & + & - & + & - & - & - & + \\
\hline 12 & $\mathrm{O} 25$ & - & + & - & - & NA & + & + & + & + & - & - & + & - & + & + & - & + \\
\hline 13 & 017 & - & + & - & + & 2 & + & - & + & + & - & - & + & - & - & + & - & - \\
\hline 14 & $\mathrm{NI}$ & - & + & - & - & NA & + & - & + & + & - & - & + & - & + & - & - & + \\
\hline 15 & 0153 & - & + & - & - & NA & + & - & + & - & - & - & - & - & + & - & - & - \\
\hline 16 & $\mathrm{O} 25$ & + & + & + & + & 3 & + & - & + & + & + & + & + & + & - & - & - & + \\
\hline 17 & Rough & - & + & - & + & - & + & - & - & - & - & - & - & - & - & + & - & - \\
\hline 18 & Rough & + & + & - & + & 3 & + & - & + & + & + & + & + & + & - & - & - & + \\
\hline 19 & $\mathrm{O} 20$ & - & - & - & - & NA & + & - & + & - & + & - & - & - & - & + & + & - \\
\hline 20 & $\mathrm{NI}$ & - & + & - & - & NA & + & - & - & - & - & - & - & - & - & + & - & - \\
\hline 21 & $\mathrm{NI}$ & - & + & - & - & NA & + & - & - & - & - & - & - & - & - & + & - & - \\
\hline 22 & $\mathrm{NI}$ & - & + & - & - & NA & + & - & - & - & - & - & - & - & - & + & - & - \\
\hline 23 & O19 & - & - & - & - & NA & + & - & + & + & + & - & - & - & - & + & - & - \\
\hline 24 & $\mathrm{O} 2$ & + & + & - & + & 3.2 & + & - & + & + & + & + & + & + & - & - & - & + \\
\hline 25 & O2 & + & + & + & + & 3.2 & + & - & + & + & + & + & + & + & - & - & - & + \\
\hline 26 & O17 & - & + & - & + & 2 & + & - & + & + & - & - & + & - & - & - & - & - \\
\hline 27 & 016 & - & + & - & + & 2 & + & - & + & + & - & - & + & - & - & - & - & + \\
\hline 28 & 0173 & - & - & - & - & NA & + & - & - & - & - & - & - & - & - & + & - & - \\
\hline 29 & O6 & + & + & - & + & 3 & + & - & - & + & - & + & - & + & - & - & - & + \\
\hline 30 & 017 & - & + & - & + & 2 & + & - & + & + & - & - & + & - & - & - & - & - \\
\hline 31 & O78 & - & - & - & - & NA & + & - & + & + & + & - & + & - & - & - & + & - \\
\hline 32 & $\mathrm{NI}$ & - & - & - & - & NA & + & - & + & + & + & - & - & - & - & + & + & - \\
\hline 33 & 075 & + & + & + & + & 2 & + & - & + & + & + & + & + & - & - & - & - & + \\
\hline 34 & $\mathrm{NI}$ & - & + & - & + & 2 & + & - & + & + & - & - & + & - & - & + & - & + \\
\hline 35 & 017 & - & + & - & + & 2 & + & - & + & + & + & - & - & - & - & + & + & - \\
\hline 36 & $\mathrm{O} 2$ & - & + & - & + & 2 & + & - & + & + & - & - & + & - & - & + & - & - \\
\hline 37 & $\mathrm{O} 2$ & - & + & - & + & 2 & + & - & + & + & + & - & - & - & - & + & + & + \\
\hline 38 & O2 & - & + & - & + & 2 & + & - & + & + & - & - & - & - & - & - & - & + \\
\hline 39 & O19 & - & - & - & - & NA & + & - & + & - & + & - & - & - & - & + & + & - \\
\hline 40 & $\mathrm{NI}$ & - & - & - & - & NA & + & - & + & - & + & - & - & - & - & - & + & - \\
\hline 41 & $\mathrm{NI}$ & - & + & - & - & NA & + & - & + & + & - & - & - & - & - & - & - & - \\
\hline 42 & 01 & - & + & - & + & 2 & + & - & + & + & - & - & + & - & - & + & - & + \\
\hline 43 & O6 & + & + & + & + & 3 & + & - & - & + & + & + & - & + & - & - & - & + \\
\hline 44 & O17 & - & + & - & + & 2 & + & + & + & + & - & - & + & - & - & + & - & - \\
\hline 45 & 017 & - & + & - & + & 2 & + & - & + & + & - & - & + & - & - & + & - & - \\
\hline 46 & O75 & + & + & - & - & NA & + & + & + & + & - & - & + & - & - & + & - & - \\
\hline 47 & O6 & + & + & + & + & 3 & + & - & - & + & + & + & - & + & - & - & - & + \\
\hline 48 & O86 & - & + & - & + & 2 & + & - & + & + & - & - & + & - & - & - & - & - \\
\hline 49 & 011 & - & + & - & + & 2 & + & - & + & + & - & - & + & - & - & - & - & - \\
\hline 50 & $\mathrm{O} 2$ & - & + & - & + & 2 & + & - & + & + & - & - & + & - & - & - & - & - \\
\hline 51 & Rough & - & + & - & - & NA & + & - & + & + & - & - & + & - & - & - & - & - \\
\hline 52 & $\mathrm{O} 1$ & - & + & - & + & 2 & + & - & + & + & + & - & - & - & - & + & + & + \\
\hline 53 & $\mathrm{O} 2$ & + & + & + & + & 3.2 & + & - & + & + & + & + & + & + & - & - & - & + \\
\hline 54 & $\mathrm{NI}$ & - & + & - & - & NA & - & + & + & + & - & - & + & - & - & + & - & - \\
\hline 55 & $\mathrm{NI}$ & - & - & - & - & NA & - & - & + & + & - & - & - & - & - & + & - & - \\
\hline 56 & 017 & - & + & - & + & 2 & + & - & + & + & - & - & + & + & - & + & - & - \\
\hline 57 & $\mathrm{NI}$ & - & - & - & + & - & - & - & + & - & - & - & - & - & - & + & - & - \\
\hline 58 & O6 & + & - & - & + & 3 & + & - & - & + & + & + & - & + & + & - & - & + \\
\hline 59 & $\mathrm{NI}$ & - & - & - & - & NA & + & - & + & + & - & - & - & - & - & + & - & - \\
\hline 60 & $\mathrm{NI}$ & - & + & - & - & NA & + & - & - & - & - & - & - & - & - & + & - & - \\
\hline
\end{tabular}

$\mathrm{NI}=$ not identifiable or not tested; $\mathrm{NA}=$ not assayed (papC negative). Capsule: $k p s M T I l ;$ adhesins and fimbriae: $\operatorname{sfaD/E}$, papC, papG (alleles $=2$ or 3 or both: 3.2), fimH, afaB/C; iron uptake: iucD, fyuA, iroN, hlyA; toxins: sat, cnf1; miscellaneous: ibeA, traT, cvaC, usp. 
serogrouping, the most common VF genes among ExPEC and invasivity, we integrated and compared basic parameters aiming to support a SEPEC profile.

However, the differential in this study is not only the three targets, but also the patients. We worked with septicemic patients who died within two days after being hospitalized and had $E$. coli isolated by hemoculture. The Hospital de Clínicas of the State University of Campinas in Brazil receives patients from the entire country. Brazil presents an interesting place to develop studies with SEPEC because of its heterogeneous population of over 190 million people. Studies focusing on SEPEC are scarce and usually only related to isolates from urosepsis. This is another important point: urosepsis differs from sepsis like UPEC differs from SEPEC. Invasivity studies have shown UPEC unable to invade Vero cells. Our SEPEC isolates had an immense invasive capacity in this cell linage.

The diversity of our isolates was indicated by the wide distribution of VF genes and serogroups. Few studies have reported the prevalence of virulence genes in sepsis $E$. coli isolates $(4,7)$. We identified a conserved virulence subgroup profile. One hundred percent of the twenty-one $\mathrm{O} 2 /$ 017/O6 strains harbored fimH, fyuA and papC. All of the strains except one, number $58(\mathrm{O} 6)$, possessed the kpsMTII gene. Individually, all $\mathrm{O} 2$ strains presented iucD and papGII; however, $50 \%$ of them were also positive for the papGIII allele. usp and iro $N$ were not $100 \%$ prevalent in this serogroup, but their prevalence was very significant.

The papGIII, hlyA, cnf1 and usp were conserved in the $\mathrm{O} 6$ strains. No $\mathrm{O} 6$ strain was $i u c D^{+}$. Significantly, iroN and $s f a D / E$ had a high prevalence. In this serogroup, number 58 strain was unique because it was kpsMTII-negative, but ibeA-positive. kpsMTII, papGll and iucD genes were $100 \%$ prevalent in the serogroup 017. sat and traT had great prevalence in this serogroup. The association of papGIII, $h l y$, and cnf1 was observed in $20 \%$ in our strains. These genes were located on a single DNA macro-restriction fragment (3).

Nearly $5 \%$ of our strains were positive for papC and negative for papG alleles I, II, and III, which is in agreement with previous reports $(2,3,13)$, suggesting that another allele may be present or that papG may be deleted.

Similarly, three strains (numbers 7, 9, and 43) presented discrepant results between the hemolytic reaction (Hly) and the PCR for the hlyA gene (Table 2). In these Hly ${ }^{+}$ hly- strains, hemolysis could have been caused by other VF rather than $\alpha$-hemolysin. Alternatively, some malfunctions in the operon hly could be another explanation. Likewise, Johnson (12) related this to the pap operon. Horizontal transfer of VFs can originate failures or incomplete transmissions $(1,2)$.
In the body fluids of warm-blooded animals, iron is almost entirely bound to the iron transport of proteins such as transferrin and lactoferrin. As a result, the free-iron concentration under these conditions is insufficient for bacterial survival and multiplication, and the pathogens depend on their ability to scavenge iron (8). Efficient iron uptake is most essential for bacteria that enter the bloodstream, and our isolates showed a significant prevalence to VF genes fyuA (81.7\%), iucD (73.8\%) and iroN (37\%). Fourteen $(23 \%)$ of the strains presented these three genes concomitantly, suggesting three independent parallel systems that probably act at different sites in the host.

Johnson and Stell (7) suggested that Yersinia high pathogenicity island may contribute as a useful target for preventive intervention. Likewise, from a practical point of view, the absence of fyuA in the urinary tract isolate would tend to exclude the risk of bacteremia in non-compromised patients. Finally, the association of papC with fyuA could be the minimal prerequisite for bacterial passage from a renal focus of infection into the bloodstream of non-compromised patients, attached to kpsMTII or another capsule or protectin.

Although classified as enteric microorganisms, many strains of $E$. coli are capable of colonizing extraintestinal tissues. In contrast to intestinal colonization, the E. coli strains that colonize other organs often generate systemic infection. The strains involved in extraintestinal diseases do not carry genes for production of enterotoxins but usually express polysaccharide capsules such as K1. Another difference between intestinal and extraintestinal $E$. coli strains involves adherence to epithelial cells. Usually, adherence of intestinal $E$. coli is mediated via adherent fimbriae, such as K99 (F5) and K88 (F4), which are coded for plasmid genes, and adhesion is mediated by the major subunit. In strains involved in human extraintestinal infection, the fimbriae are encoded by chromosomal genes and composed of a major subunit and several minor subunits, which influence binding abilities and are responsible for specific adhesion (14).

The group of S-fimbria is interesting because it contains several types of fimbriae with different adherence specificities that are produced by bacteria that cause a variety of clinical symptoms in mammals and poultry. Nevertheless, all strains $s f a D / E^{+}(18.3 \%)$ are also $p a p C^{+}$and fimH $H^{+}$. S-fimbriae (sfaD/E) did not appear to act as an essential VF because the total prevalence of papC (65\%) and $\mathrm{fimH}(95 \%)$ were much higher and the results are consistent with several other studies, including afaD/E $(6.7 \%)(7)$. It is possible that other VF genes related to ExPEC adhesins such as bmaE, focG, gafD, and iha are present in our isolates. 
ExPEC cause extraintestinal diseases in human beings, much as APEC does in birds. APEC-related genes used in this study have been found on a large, conjugative R plasmid, known as pTJ100, which encodes aerobactin and ColV production, from a virulent avian $E$. coli strain (15). cvaC, iroN and traT are examples of these genes, and, in this study, they showed lower prevalence compared with APEC (16). However, we found a significant prevalence of traT $(50 \%)$ and $\operatorname{iroN}(37 \%)$, inferring a relationship between ExPEC and APEC. Additionally, Johnson et al. (15) isolated $E$. coli strains from retail chicken products that had virulence profiles similar to EXPEC isolates. Guyer et al. (17) concluded that these strains represent potential human pathogens.

Another gene described in APEC and ExPEC is ibeA, a gene encoding a known VF of $E$. colistrains responsible for neonatal meningitis in humans; it is able to invade brain microvascular epithelial cells (9). Four of our isolates possess ibeA (6.7\%). These data concur with other studies in ExPEC (4) and APEC (16). However, Germon et al. (9) reported that $i b e A$ is positively associated with 088,018 and $\mathrm{O} 2$ strains, but our data did not demonstrate this association.

In sepsis, bacteria need VFs that help the organisms avoid and/or subvert host defense mechanisms. The kpsMTII (81.3\%) capsular gene and traT could be acting in this manner, which explains the high prevalence in our isolates. Interestingly, of the 11 negative strains of $k p s M T I I$, 9 possessed traT.

The significant prevalence of sat (55\%) and usp (37\%) point to UPEC, especially urosepsis. Sat is a toxin and can injure host cells and tissues (17), like cytotoxic necrotizing factor 1 (cnf1) and hemolysin ( $h l y A)$, inciting a noxious inflammatory response, thereby causing clinical disease.

We chose Vero cells to test invasivity in our isolates because they are a good representation of the urinary tract. The kidneys could be a possible entry point of $E$. coli into blood circulation. It is interesting that invasivity was almost $100 \%$, which is contrary to some data in scientific literature, such as Peerbooms et al. (18). They tested Vero cell invasiveness for a group of Proteus mirabilis strains isolated from the urinary tract and feces and for a limited group of urinary isolates of $E$. coli. All of the $P$. mirabilis strains tested were capable of cell invasion, whereas none of the $E$. coli strains were.

These results indicate that each step in the infection process can be mediated by a number of alternative VFs, and each strain may have a unique combination of these factors. This assortment of virulence genes is made feasible by the variety of genetic factors contributing to genome plasticity, such as plasmids, phages, and transposable elements (1). Johnson et al. (2) concluded that the considerable diversity of VF profiles noted at every level within the phylogenetic tree suggests that many different pathways can lead to extraintestinal virulence in $E$. coli and that the evolution of ExPEC, which involves extensive horizontal transmission of VFs and continuous remodeling of PAls, is a highly active and ongoing process.

The fact that extant $E$. coli strains vary so much in their genome contents indicates that this mix-and-match combinational approach has been a successful evolutionary strategy for this species, which can colonize many different tissues and hosts (1). Comparison of strains causing similar types of diseases is essential for the identification of shared traits, which are important for pathogenicity and for the study of their conservation, transfer, and evolution.

In this investigation, we have characterized a collection of $E$. coli sepsis isolates from Brazil with respect to their VFs and serogroup profile. The evidence showed that not many VFs are common to different SEPEC strains. This is surprising since phenotypically different strains cause the same disease. On the other hand, the vast majority of them were able to invade Vero cells. Our findings imply that different strains can use different factors with similar roles in the various stages of the infection process.

\section{Acknowledgments}

The authors wish to thank Ana Stella Menegon Degrossoli for technical assistance and David Elieff for helping with the English language review. We are grateful to the Microbiology Laboratory of the Hospital de Clínicas de Campinas, Universidade Estadual de Campinas, São Paulo, Brasil, for kindly providing the strains.

\section{References}

1. Mokady D, Gophna U, Ron EZ. Extensive gene diversity in septicemic Escherichia coli strains. J Clin Microbiol 2005; 43: 66-73.

2. Johnson JR, O'Bryan TT, Kuskowski M, Maslow JN. Ongoing horizontal and vertical transmission of virulence genes and papA alleles among Escherichia coli blood isolates from patients with diverse-source bacteremia. Infect Immun 2001; 69: 5363-5374.

3. Bingen-Bidois $\mathrm{M}$, Clermont $\mathrm{O}$, Bonacorsi S, Terki M, Brahimi $\mathrm{N}$, Loukil $\mathrm{C}$, et al. Phylogenetic analysis and prevalence of 
urosepsis strains of Escherichia coli bearing pathogenicity island-like domains. Infect Immun 2002; 70: 3216-3226.

4. Johnson JR, Russo TA, Tarr PI, Carlino U, Bilge SS, Vary $\mathrm{JC} \mathrm{Jr}$, et al. Molecular epidemiological and phylogenetic associations of two novel putative virulence genes, iha and iron (E. coli), among Escherichia coli isolates from patients with urosepsis. Infect Immun 2000; 68: 3040-3047.

5. Daigle F, Harel J, Fairbrother JM, Lebel P. Expression and detection of pap-, sfa-, and afa-encoded fimbrial adhesin systems among uropathogenic Escherichia coli. Can J Microbiol 1994; 40: 286-291.

6. Yamamoto S, Terai A, Yuri K, Kurazono H, Takeda Y, Yoshida O. Detection of urovirulence factors in Escherichia coli by multiplex polymerase chain reaction. FEMS Immunol Med Microbiol 1995; 12: 85-90.

7. Johnson JR, Stell AL. Extended virulence genotypes of Escherichia coli strains from patients with urosepsis in relation to phylogeny and host compromise. J Infect Dis 2000; 181: 261-272.

8. Gophna U, Oelschlaeger TA, Hacker J, Ron EZ. Yersinia $\mathrm{HPI}$ in septicemic Escherichia coli strains isolated from diverse hosts. FEMS Microbiol Lett 2001; 196: 57-60.

9. Germon $\mathrm{P}$, Chen YH, He L, Blanco JE, Bree A, Schouler C, et al. ibeA, a virulence factor of avian pathogenic Escherichia coli. Microbiology 2005; 151: 1179-1186.

10. Vandekerchove D, Vandemaele F, Adriaensen C, Zaleska $M$, Hernalsteens JP, De Baets L, et al. Virulence-associated traits in avian Escherichia coli: comparison between isolates from colibacillosis-affected and clinically healthy layer flocks. Vet Microbiol 2005; 108: 75-87.
11. Nakano M, Yamamoto S, Terai A, Ogawa O, Makino SI, Hayashi $\mathrm{H}$, et al. Structural and sequence diversity of the pathogenicity island of uropathogenic Escherichia coli which encodes the USP protein. FEMS Microbiol Lett 2001; 205: 71-76.

12. Johnson JR. papG alleles among Escherichia coli strains causing urosepsis: associations with other bacterial characteristics and host compromise. Infect Immun 1998; 66: 4568-4571.

13. Otto G, Magnusson M, Svensson M, Braconier J, Svanborg C. pap genotype and $\mathrm{P}$ fimbrial expression in Escherichia coli causing bacteremic and nonbacteremic febrile urinary tract infection. Clin Infect Dis 2001; 32: 1523-1531.

14. Babai R, Stern BE, Hacker J, Ron EZ. New fimbrial gene cluster of S-fimbrial adhesin family. Infect Immun 2000; 68: 5901-5907.

15. Johnson TJ, Giddings CW, Horne SM, Gibbs PS, Wooley $\mathrm{RE}$, Skyberg J, et al. Location of increased serum survival gene and selected virulence traits on a conjugative $R$ plasmid in an avian Escherichia coli isolate. Avian Dis 2002; 46: 342-352.

16. Rodriguez-Siek KE, Giddings CW, Doetkott C, Johnson TJ, Nolan LK. Characterizing the APEC pathotype. Vet Res 2005; 36: 241-256.

17. Guyer DM, Henderson IR, Nataro JP, Mobley HL. Identification of sat, an autotransporter toxin produced by uropathogenic Escherichia coli. Mol Microbiol 2000; 38: 53-66.

18. Peerbooms PG, Verweij AM, Maclaren DM. Vero cell invasiveness of Proteus mirabilis. Infect Immun 1984; 43: 10681071. 